\title{
Risk and protective factors for the health of primary care-givers of children with autism spectrum disorders or intellectual disability: a narrative review
}

\author{
Jenny Fairthorne ${ }^{1,2,3^{*}}$, Yuka Mori ${ }^{3}$ and Helen Leonard ${ }^{3}$
}

*Correspondence: Jenny.Fairthorne@york.ac.uk

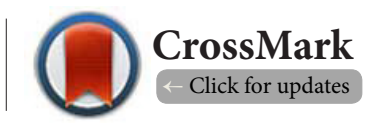

'British Columbia Children's Hospital Research Institute, BC Children's Hospital, 4480 Oak St Vancouver, BC V6H 3V4, Canada. ${ }^{2}$ Department of Health Sciences, University of York, Heslington, York, YO10 3DD, UK.

${ }^{3}$ Telethon Kids Institute, University of Western Australia, Perth, Australia.

\begin{abstract}
Background: Primary care-givers of children with autism spectrum disorder (ASD) or intellectual disability (ID) have poorer health and quality of life than primary care-givers of typically developing children. We aimed to review original research which described factors impacting the health of primary care-givers of children with ASD or ID and to discuss how these factors might influence care-giver health.

Methods: We searched electronic databases and retrieved 33 relevant research articles.

Results: Factors impacting primary care-giver health included child behaviour, support and diagnostic issues. We explain how these factors might impact care-giver health and how they might act differentially in care-givers of children with different types of ASD or ID. Interventions to moderate one or more risk factors could pave the way for improved primary care-giver health.
\end{abstract}

Keywords: Autism, intellectual disability, mothers; care-givers, health, review

\section{Introduction}

A person with autism spectrum disorder (ASD) has a life-long neuro-developmental disability which is diagnosed by severe deficits in social communication and interaction, along with restricted or repetitive behaviour and interests [1]. Intellectual disability (ID) is diagnosed in persons with an IQ below 70 along with impairments in adaptive functioning both of which must be present before 18 years [1]. The prevalence of ASD varies between countries with a median of about 60 per 10,000 [2] while the prevalence of ID is higher at 104 per 10,000 [3].

There is a general consensus among researchers that Quality of Life $(\mathrm{QoL})$ incorporates measures of physical functioning, mental health and engagement in social interaction [4]. A plethora of research has identified poorer health and QoL in the primary care-givers (here after referred to as care-givers) of children with ASD or ID than in other care-givers [5-11]. For example, using linked data, we demonstrated that in the years after their child's birth, mothers of children with ASD or ID had higher rates of psychiatric disorders than other mothers $[9,10]$. Furthermore, we also identified health differentials in mothers of children with different sub-types of ASD or ID [12]. Using the 12 Item Short Form Health Survey (SF-12), parents of children with ASD without ID were also reported as experiencing lower QoL and more stress than parents of children with ASD with ID [7]. Moreover, mothers of children with ASD had more self-reported anxiety and depression than mothers of children with Down syndrome [13]. Care-giver health issues challenge a family's QoL and may impact the care and healthy development of all children within the family $[\mathbf{1 4 , 1 5 ]}$. Identifying risk factors for care-givers of children with a particular developmental disorder would enable the identification of groups whose health is especially vulnerable. In addition, identifying both risk and protective factors would enable a better understanding of the health trajectories of care-givers of children with ASD or ID. In such ways, informed interventions and services might be developed to assist in optimising care-giver health. Therefore, we aimed to review the research literature in the area of care-giver health, ASD and ID to locate original research which described risk and/or protective factors for health in care-givers of children with ASD or ID. Our second aim was to discuss how

(C) 2018 Fairthorne et al; licensee Herbert Publications Ltd. This is an Open Access article distributed under the terms of Creative Commons Attribution License (http://creativecommons.org/licenses/by/3.0). This permits unrestricted use, distribution, and reproduction in any medium, provided the original work is properly cited. 
Fairthorne et al., Journal of Autism 2018,

http://www.hoajonline.com/journals/pdf/2054-992X-5-1.pdf

doi: 10.7243/2054-992X-5-1

these factors might influence care-giver health.

\section{Methods of the review}

We searched the electronic databases, Web of Knowledge, Medline, Scopus and Google Scholar, using combinations of the following groups of search terms associated with ASD and ID along with care-giver health. These included terms which are related to:

- $\quad$ ASD, ID or sub-groups such as autism, autistic, pervasive development disorder, intellectual disability, mental retardation, disability, disabled, Down, Asperger;

- Health such as health, depression, physical, mental, psychiatric, psychological, phenotype, quality of life, well-being;

- Care-givers such as mothers, parents, care-givers.

We included an original paper in our review if it:

- Was published between 1st January 1990 and 31st December, 2016

- Described original research in a peer-reviewed article which was written in English

- Used a study population of 15 persons or more

- Described a risk or protective factor for poorer health in mothers, parents or care-givers of a child with ASD or ID or a sub-type (such as ASD with ID or Down syndrome). Using research $[16,17]$, we developed a simple and objective five-level tool to assess the strength of evidence provided by the papers in this review (Table 1).

\section{Results}

We retained 33 articles. Table 2 summarizes each paper, provides an assessment of the quality of evidence, methods of data collection and recruitment, country of origin, study population, comparison group and the strength of the assessed relationship or a key result.

\section{Discussion}

In the first section, we discuss the papers retrieved for the review according to the reported risk or protective factors for poorer care-giver health or QoL. In the second section, we examine care-giver health disparities according to the child's diagnosis and the likely impact of particular risk or protective factors. The final section examines how risk and protective factors might impact the health of care-givers of children with ASD or ID.

\section{Challenging child behavior}

Challenging child behaviour, the most commonly identified risk factor for poorer care-giver health and QoL outcomes, was reported in ten of the 33 studies. Researchers provided substantial evidence that the social impact of the child's disability [18] and challenging behaviours [19-25] in children with ASD or ID was associated with poorer care-giver mental health. At the same level of evidence, challenging child behaviours in children with ID were related to poorer care-giver physical health in subsequent years [26]. The total score for the Jenkins Behaviour Questionnaire was higher in children with care-givers having two or more symptoms of depression $(p=0.004)$ [27]. Symptoms of anxiety $(p=0.06)$ and stress $(p=0.08)$ were related also to challenging child behaviour though the associations did not reach statistical significance. These associations were predominantly due to issues of discipline (control, tantrums, demanding attention) and disturbances in sleep [27].

Challenging child behaviours had a negative effect on care-giver and family QoL. One research group administered the Parental-Developmental Disorders-Quality of Life Scale (Par-DD-QoL) to the parents of children with ASD and provided weak evidence that challenging child behaviour was an independent risk factor for poorer QoL [28]. Results from a qualitative study were similar [24].

\section{Level of support}

Support networks were associated with improved care-giver health and QoL in families of a child with ASD or ID. For example, substantial evidence indicated that family support reduced care-giver burden in families with a child with ASD $[20,29]$ and that family and neighbourhood support was associated with improved parental mental health $[20,30,31]$ and reduced parental aggravation or irritability [32]. Further substantial evidence [33] [as classified by our descriptors on Table 1] was provided using the Multidimensional Scale of Perceived Social Support (MSPSS) which indicated that greater social support was associated with lower levels of individual distress (apart from anxiety and depression) in the families of children with ASD [25]. Alternatively, substantial evidence demonstrated that mothers of children with ASD had poorer mental health than other mothers but no less social support for parenting [34].

Higher levels of support had a similar favourable effect

Table 1. Assessment of level of evidence

\begin{tabular}{llll}
\hline Level of evidence & Narrow descriptor & Broader descriptor & Description of study \\
\hline 5 & Strong & Substantial & Validated scores \& magnitude of effect assessed \\
4 & Moderate & Validated scores but no magnitude of effect assessed \\
3 & Mild & Magnitude of effect assessed but no validated scores \\
\hline 2 & Weak & Less substantial & No validated scores or assessment of magnitude of effect \\
1 & Indication only & & Descriptive study \\
\hline
\end{tabular}


Fairthorne et al., Journal of Autism 2018,

http://www.hoajonline.com/journals/pdf/2054-992X-5-1.pdf

doi: 10.7243/2054-992X-5-1

Table 2. Details of papers included in the review.

\begin{tabular}{|c|c|c|c|c|c|c|c|c|c|}
\hline \multirow[t]{2}{*}{ Study } & \multirow[t]{2}{*}{ Level } & \multirow{2}{*}{$\begin{array}{l}\text { Outcome } \\
\text { measurement }^{*}\end{array}$} & \multirow[t]{2}{*}{ Recruitment } & \multirow{2}{*}{$\begin{array}{l}\text { Country } \\
\text { of origin }\end{array}$} & \multirow[t]{2}{*}{ Study population } & \multirow{2}{*}{$\begin{array}{l}\text { Comparison } \\
\text { groups }\end{array}$} & \multicolumn{2}{|c|}{ Variables investigated } & \multirow{2}{*}{$\begin{array}{l}\text { Strength of relationship (1 vs } 2 \text { ) or key } \\
\text { result(s) }\end{array}$} \\
\hline & & & & & & & 1 & 2 & \\
\hline $\begin{array}{l}\text { 1. Baghdadli, } 2014 \\
\text { (28) }\end{array}$ & 2 & $\begin{array}{l}\text { Parental-DD-QoL } \\
\text { Scale }\end{array}$ & $\begin{array}{l}\text { ASD evaluation } \\
\text { clinics }\end{array}$ & France & $\begin{array}{l}152 \text { mothers } \\
\text { of children with } \\
\text { ASD, } 182 \text { others }\end{array}$ & $\begin{array}{l}\text { ASD, severe } \\
\text { chronic } \\
\text { diseases }\end{array}$ & $\begin{array}{l}\text { Child behaviour } \\
\text { (ABC) }\end{array}$ & Parental QoL & $\begin{array}{l}\text { Child behaviour was an independent } \\
\text { risk factor for parental QoL }\end{array}$ \\
\hline \multirow[t]{2}{*}{ 2. Bourke, 2008 (19) } & 5 & SF-12 & Service providers & Australia & 250 cases & DS, pop norm & $\begin{array}{l}\text { Independence of } \\
\text { learning skills } \\
\text { (WeeFIM-m) }\end{array}$ & Maternal PH & $\begin{array}{l}\text { Mothers of children with Independence } \\
\text { of leaming skills had better PH than } \\
\text { mothers of children without }\end{array}$ \\
\hline & & & & & & & $\begin{array}{l}\text { Child behaviour } \\
\text { (DBC) }\end{array}$ & Maternal health & $\begin{array}{l}\text { Negative association of PH \& MH with } \\
\text { poorer child behaviour (PCS: } \\
\text { coefficient }=-0.3, \text { MCS: coefficient=- } 0.8 \text { ) }\end{array}$ \\
\hline 3. Bromley, 2004 (20) & 5 & GHQ-12 & Service providers & UK & 68 mothers & ASD & $\begin{array}{l}\text { - Child behaviour } \\
\text { (DBC) } \\
\text { - Family support } \\
\text { (FSI) }\end{array}$ & $\begin{array}{l}\text { Maternal } \\
\text { distress }\end{array}$ & $\begin{array}{l}\text { Maternal distress was associated with } \\
\text { poor child behaviour \& lower family } \\
\text { support }\end{array}$ \\
\hline 4. Caldwell, 2008 (37) & 5 & SF-36 & Service providers & US & 1,400 cases & DD, pop norm & $\begin{array}{l}\text { Access to services } \\
\text { (FSI modified) }\end{array}$ & Maternal PH & $\begin{array}{l}\text { Negative association of } \mathrm{PH} \text { with poorer } \\
\text { access to services (coefficient:-0.2) }\end{array}$ \\
\hline 5. Cantwell, 2014 (38) & 5 & $\begin{array}{l}\text { Physical Health } \\
\text { Questionnaire }\end{array}$ & University/other & Ireland & 109 cases & $\mathrm{DD}, \mathrm{TD}$ & $\begin{array}{l}\text { Support } \\
\text { (SFS) }\end{array}$ & Maternal PH & $\begin{array}{l}\text { No direct relationship between PH\& } \\
\text { support needs } \\
\text { Effects of stress on PH moderated by } \\
\text { social support }\end{array}$ \\
\hline 6. Corrice, $2009(43)$ & 4 & $\begin{array}{l}\text { Subjective Well- } \\
\text { being, Child- } \\
\text { Related Scale }\end{array}$ & $\begin{array}{l}\text { Service providers \& } \\
\text { community }\end{array}$ & US & 120 mothers & $\mathrm{DS}, \mathrm{DD}$ not DS & $\begin{array}{l}\text { Personal rewards } \\
\text { (TDRWQ) }\end{array}$ & $\begin{array}{l}\text { Maternal well- } \\
\text { being }\end{array}$ & $\begin{array}{l}\text { DS mothers had higher average reward } \\
\& \text { subjective well-being compared to } \\
\text { mothers of children with other DDs. }\end{array}$ \\
\hline 7. Dardas, $2014(41)$ & 3 & WHOQOL-BREF & Community & Jordan & 184 parents & $A D$ & Income & Parental QoL & $\begin{array}{l}\text { Correlation } \approx 0.2 \text { between parental } \\
\text { income } \& Q \circ L\end{array}$ \\
\hline 8. De Grace, 2014 (46) & 1 & Interviews & Community & US & 7 families & ASD & $\begin{array}{l}\text { Family } \\
\text { experiences }\end{array}$ & - & Not applicable \\
\hline $\begin{array}{l}\text { 9. Eisenhower, } 2013 \\
\text { (26) }\end{array}$ & 5 & $\begin{array}{l}\text { Single-item } \\
\text { measurefor PH }\end{array}$ & Service providers & US & 116 cases & $\mathrm{TD}, \mathrm{DD}$ & $\begin{array}{l}\text { Child behaviour } \\
\text { (CBC) }\end{array}$ & Maternal PH & $\begin{array}{l}\text { Poorer child behaviour was associated } \\
\text { with poorer } \mathrm{PH} \text { in DD group. }\end{array}$ \\
\hline $\begin{array}{l}\text { 10. Emerson, } 2003 \\
\text { (18) }\end{array}$ & 5 & GHQ-12 & Survey participants & UK & $\begin{array}{l}245 \text { case mothers, } \\
9,481 \text { control } \\
\text { mothers }\end{array}$ & ID, No ID & $\begin{array}{l}\text { Social impact of } \\
\text { child's difficulties } \\
\text { (SDQ) }\end{array}$ & Maternal MH & $\begin{array}{l}\text { No direct relationship between the } \\
\text { social impact of child's difficulties \& MH }\end{array}$ \\
\hline $\begin{array}{l}\text { 11. Emerson, } 2010 \\
\text { (39) }\end{array}$ & 3 & Kessler-6 & Previous study & UK & $\begin{array}{l}\text { More than } 15,000 \\
\text { parents }\end{array}$ & Cognitive delay & $\begin{array}{l}\text { Severe delay, Less } \\
\text { severe delay, TD } \\
\text { (BBCS \& NVS) }\end{array}$ & $\begin{array}{l}\text { Maternal } \\
\text { psychiatric } \\
\text { disorder (MPD) }\end{array}$ & $\begin{array}{l}\text { - RR=1.5/1.2 for MPD \& child with } \\
\text { severe/less severe delay at } 5 \\
\text { yearscompared to mothers of TD child } \\
\text { - Low SES associated with having MPD }\end{array}$ \\
\hline 12. Estes, 2009 (21) & 4 & $\begin{array}{l}\text { Questionnaire } \\
\text { assessing } \\
\text { stress }\end{array}$ & Service providers & US & $\begin{array}{l}\text { 51 ASD, } 22 \text { DD no } \\
\text { ASD }\end{array}$ & ASD, DD no ASD & $\begin{array}{l}\text { Child behaviour } \\
\text { (ABC) }\end{array}$ & Maternal stress & $\begin{array}{l}\text { Mothers of children with ASD had } \\
\text { higher average stress \& children with } \\
\text { ASD had poorer child behaviour. }\end{array}$ \\
\hline 13. Firth , $2013(22)$ & 4 & PSS & $\begin{array}{l}\text { Service providers \& } \\
\text { community }\end{array}$ & Australia & 109 parents & ASD & $\begin{array}{l}\text { Child behaviour } \\
\text { (NCBRF) }\end{array}$ & $\begin{array}{l}\text { Parental } \\
\text { distress }\end{array}$ & $\begin{array}{l}\text { Positive association of distress with } \\
\text { poorer child behaviour (coefficient } \\
\approx 0.3 \text { ) }\end{array}$ \\
\hline 14. Harvey, 1997 (27) & 4 & $\begin{array}{l}\text { Items from DSSI } \\
\& \text { GHQ- } 28\end{array}$ & Service providers & Australia & 65 cases & $\mathrm{DD}, \mathrm{TD}$ & $\begin{array}{l}\text { Child behaviour } \\
\text { (Behaviour } \\
\text { Checklist of } \\
\text { Jenkins) } \\
\end{array}$ & $\begin{array}{l}\text { Maternal } \\
\text { depression }\end{array}$ & $\begin{array}{l}\text { No association of depression with child } \\
\text { behaviour }\end{array}$ \\
\hline 15. Herring, $2006(23)$ & 4 & GHQ-28 & Child clinic & Australia & 123 parents & $P D D, D D \sim P D D$ & $\begin{array}{l}\text { Child behaviour } \\
\text { (DBC-P) }\end{array}$ & Parental MH & $\begin{array}{l}\text { Poorer maternal MH was associated } \\
\text { with poorer child behaviour (regression } \\
\text { coefficient } \approx 10.11 \text { ). }\end{array}$ \\
\hline 16. Hoppes, $1990(42)$ & 3 & $\begin{array}{l}\text { Ad hoc } \\
\text { questionnaire }\end{array}$ & Service providers & US & 38 mothers & ASD, DS & $\begin{array}{l}\text { Child's disability } \\
\text { group }\end{array}$ & $\begin{array}{l}\text { Maternal } \\
\text { gratification } \\
\text { (ad hoc } \\
\text { questionnaire) } \\
\end{array}$ & $\begin{array}{l}\text { Mothers from ASD group had less } \\
\text { gratification than DS mothers } \\
\text { - Improved MH with more support }\end{array}$ \\
\hline $\begin{array}{l}\text { 17. Ingersoll, } 2011 \\
(52)\end{array}$ & 3 & CES-D & ASD web site & US & 90 case mothers & ASD & $\begin{array}{l}\text { BAO, ASQ } \\
\text { Autism severity } \\
\text { (Autism Behavior } \\
\text { Checklist) }\end{array}$ & $\begin{array}{l}\text { Maternal } \\
\text { depressed } \\
\text { mood }\end{array}$ & $\begin{array}{l}\text { Increased depression attributed to both } \\
\text { stress of child \&BAP. }\end{array}$ \\
\hline $\begin{array}{l}\text { 18. Ingersoll, } 2011 \\
\text { (30) }\end{array}$ & 4 & CES-D & ASD web-site & US & 149 parents & ASD & $\begin{array}{l}\text { Social support } \\
\text { (MOS-SSS) }\end{array}$ & $\begin{array}{l}\text { Parental } \\
\text { depression }\end{array}$ & $\begin{array}{l}\text { Correlation } \approx-0.5 \text { between social } \\
\text { support \& parental depression }\end{array}$ \\
\hline \multirow[t]{2}{*}{ 19. Ji, 2014 (29) } & 4 & SF-36 & Service providers & China & 273 care-givers & ASD & $\begin{array}{l}\text { Carer load (Care- } \\
\text { giver Burden } \\
\text { Index) }\end{array}$ & $\begin{array}{l}\text { Care-giver } \\
\text { health }\end{array}$ & $\begin{array}{l}\text { Negative association of increased } \\
\text { burden of care \& care-giver health } \\
\text { (PCS: coefficient=-0.4, MCS: } \\
\text { coefficient=-0.59). }\end{array}$ \\
\hline & & & & & & & $\begin{array}{l}\text { Social support } \\
\text { (MSPSS) }\end{array}$ & $\begin{array}{l}\text { Care-giver } \\
\text { health }\end{array}$ & $\begin{array}{l}\text { Positive association of care-giver health } \\
\text { with increased social support (PCS: } \\
\text { coefficient }=0.25 \text {, MCS: coefficient=- } \\
0.24 \text { ). }\end{array}$ \\
\hline 20. Kelso, 2005 (45) & 1 & Interviews & $\begin{array}{l}\text { Schools, support } \\
\text { groups }\end{array}$ & Australia & $\begin{array}{l}26 \text { carers of } \\
\text { children with } \\
\text { disability }\end{array}$ & Not applicable & 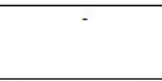 & - & - \\
\hline 21. Lach, 2009 (63) & 5 & $\begin{array}{l}\text { Shortversion of } \\
\text { CESD }\end{array}$ & Previous study & Canada & 9,467 carers & $\begin{array}{l}\text { Carers of child } \\
\text { with neuro dis } \\
\text { \& behaviour } \\
\text { problems, } \\
\text { Carers of } \\
\text { children w/out }\end{array}$ & $\begin{array}{l}\text { Disability group } \\
\text { (Health Utilities } \\
\text { Index) }\end{array}$ & $\begin{array}{l}\text { Carer } \\
\text { depression }\end{array}$ & $\begin{array}{l}\text { Increased depression in carers of } \\
\text { children with neurological disorders. }\end{array}$ \\
\hline 22. Lenhard, 2005 (47) & 3 & $\begin{array}{l}\text { - } \quad \text { Ad hoc } \\
\text { questionnaire } \\
\text { - S-T-A-I }\end{array}$ & Service providers & Germany & $\begin{array}{l}411 \text { DS, } 66 \text { ID of } \\
\text { unknown cause \& } \\
69 \text { TD mothers }\end{array}$ & $\begin{array}{l}\text { Mothers of } \\
\text { children with } \\
\text { DS, ID of } \\
\text { unknown cause, } \\
\text { TD }\end{array}$ & Disability group & $\begin{array}{l}\text { Maternal } \\
\text { anxiety }\end{array}$ & $\begin{array}{l}\text { Mothers with no diagnosis had higher } \\
\text { levels of anxiety than DS mothers who } \\
\text { had higher levels of anxiety than } \\
\text { mothers of TD children. }\end{array}$ \\
\hline 23. Lingen, 2015 (48) & 5 & $\begin{array}{l}\text { Ulm Quality of } \\
\text { Life Inventory for } \\
\text { Parents }\end{array}$ & $\begin{array}{l}\text { Parents of patients } \\
\text { with DD/ID at } \\
\text { University Hospital }\end{array}$ & Denmark & 95 mothers & $\begin{array}{l}\text { Mothers of } \\
\text { children with } \\
\text { diagnoses, } \\
\text { mothers of } \\
\text { children } \\
\text { without }\end{array}$ & $\begin{array}{l}\text { Etiological versus } \\
\text { no etiological } \\
\text { diagnosis for } \\
\text { DD/ID after } \\
\text { genetic testing } \\
\text { (aCGH) }\end{array}$ & Maternal QoL & $\begin{array}{l}\text { Maternal QoL score was } 20.2 \text { percentile } \\
\text { rank scales higher in mothers of } \\
\text { children with diagnoses compared to } \\
\text { mothers of children with no diagnoses. }\end{array}$ \\
\hline
\end{tabular}


Fairthorne et al., Journal of Autism 2018,

Continuation of Table 2.

\begin{tabular}{|c|c|c|c|c|c|c|c|c|c|}
\hline Study & Level & $\begin{array}{l}\text { Outcome } \\
\text { measurement* }\end{array}$ & Recruitment & $\begin{array}{l}\text { Country } \\
\text { of origin }\end{array}$ & Study population & $\begin{array}{l}\text { Comparison } \\
\text { groups }\end{array}$ & Variables i & estigated & $\begin{array}{l}\text { Strength of relationship (1 vs 2) or key } \\
\text { result(s) }\end{array}$ \\
\hline \multirow[t]{2}{*}{$\begin{array}{l}\text { 24. Llewellyn, } 2010 \\
\text { (35) }\end{array}$} & \multirow[t]{2}{*}{5} & \multirow[t]{2}{*}{ SF-12 } & \multirow[t]{2}{*}{ Service providers } & \multirow[t]{2}{*}{ Australia } & \multirow[t]{2}{*}{$\begin{array}{l}64 \text { older parent } \\
\text { carers ( }>60 \text { years) }\end{array}$} & \multirow{2}{*}{$\begin{array}{l}\text { Parents of } \\
\text { children with ID } \\
\text { vs pop norm }\end{array}$} & Age group & Care-giver $\mathrm{MH}$ & $\begin{array}{l}\text { Amongst carers over } 54 \text { years, mental } \\
\text { health improved with age. }\end{array}$ \\
\hline & & & & & & & Partner & Care-giver PH & Carers with partners had better PH. \\
\hline \multirow[t]{2}{*}{ 25. McStay, $2014(24)$} & \multirow[t]{2}{*}{4} & \multirow[t]{2}{*}{ PSI } & \multirow[t]{2}{*}{ Autism Centre } & \multirow[t]{2}{*}{ Australia } & \multirow[t]{2}{*}{$\begin{array}{l}196 \text { parents \& } \\
\text { population norms }\end{array}$} & \multirow{2}{*}{$\begin{array}{l}\text { Parents of } \\
\text { children with } \\
\text { ASD }\end{array}$} & $\begin{array}{l}\text { Child behaviour } \\
\text { (BASC-2) }\end{array}$ & \multirow[t]{2}{*}{$\begin{array}{l}\text { Maternal \& } \\
\text { paternal stress }\end{array}$} & \multirow{2}{*}{$\begin{array}{l}\text { Better child behaviour \& increasing } \\
\text { marital support predicted reduced } \\
\text { maternal stress }\end{array}$} \\
\hline & & & & & & & $\begin{array}{l}\text { Marital support } \\
\text { (DAS) }\end{array}$ & & \\
\hline 26. Montes, 2007 (34) & 3 & $\begin{array}{l}\text { Maternal overall } \\
\text { health measured } \\
\text { by } 1 \text { question }\end{array}$ & Previous study & US & 61,772 mothers & $\begin{array}{l}\text { Mothers of } \\
\text { children with } \\
\text { ASD, TD }\end{array}$ & Disability group & Maternal MH & $\begin{array}{l}\text { Having a child with ASD was associated } \\
\text { with poorer MH. }\end{array}$ \\
\hline 27. Mugno, 2007 (33) & 5 & WHOQOL-BREF & Service providers & Italy & $\begin{array}{l}135 \text { cases, } 42 \\
\text { controls }\end{array}$ & $\begin{array}{l}\text { Mothers of } \\
\text { children with } \\
\text { PDD, TD }\end{array}$ & $\begin{array}{l}\text { Disability group } \\
\text { (DSM4-TR) }\end{array}$ & QoL & $\begin{array}{l}\text { Controls had better QoL than parents of } \\
\text { children with PDD. }\end{array}$ \\
\hline 28. Olsson, 2001 (36) & 5 & $\mathrm{BDI}$ & $\begin{array}{l}\text { - Service provid- } \\
\text { erss (for cases) } \\
\text { - Randomised } \\
\text { mailing (for } \\
\text { controls) } \\
\end{array}$ & Sweden & $\begin{array}{l}216 \text { case, } 214 \\
\text { control mothers }\end{array}$ & $\begin{array}{l}\text { Mothers of } \\
\text { children with ID } \\
\text { with/w/out ASD } \\
\text { \& controls }\end{array}$ & Disability group & $\begin{array}{l}\text { Maternal } \\
\text { depression }\end{array}$ & $\begin{array}{l}\text { - Mothers from ASD group had less } \\
\text { gratification than DS mothers } \\
\text { - Improved MH with more support }\end{array}$ \\
\hline \multirow[t]{2}{*}{ 29. Paynter, 2013 (25) } & 5 & PSI-SF & Community & Australia & 43 parents & ASD & $\begin{array}{l}\text { Child behaviour } \\
\text { (SDQ) }\end{array}$ & Parental stress & $\begin{array}{l}\text { Positive association of stress \& poorer } \\
\text { child behaviour (coefficient } \approx 0.65 \text { ) }\end{array}$ \\
\hline & & & & & & & $\begin{array}{l}\text { Support } \\
\text { (MSPSS) }\end{array}$ & Parental stress & $\begin{array}{l}\text { Negative association of support \& stress } \\
\text { (coefficient } \approx-0.60 \text { ) }\end{array}$ \\
\hline 30. Schieve, 2011(32) & 3 & $\begin{array}{l}\text { Aggravation in } \\
\text { Parenting Scale } \\
\text { (APS) }\end{array}$ & Previous study & US & 872 cases & ASD, TD & $\begin{array}{l}\text { ASD } \\
\text { TD }\end{array}$ & $\begin{array}{l}\text { Parental } \\
\text { aggravation }\end{array}$ & $\begin{array}{l}\text { Parenting a child with ASD was } \\
\text { associated with more aggravation. }\end{array}$ \\
\hline 31. Singhi, $1990(40)$ & 3 & SBS & Service providers & India & 50 case parents & ID, TD & $\begin{array}{l}\text { ID, TD (Used } 1 \text { or } \\
2 \text { of items below } \\
\text { to assess IQ) } \\
\text { GDT, SFB, MIS, } \\
\text { VSMS }\end{array}$ & Social burden & $\begin{array}{l}\text { ID parents had higher burden: } I D=14.6 \text {, } \\
\text { Control=0.72. }\end{array}$ \\
\hline 32. Werner, 2015 (44) & 4 & AFS & Convenience sample & Israel & 171 care-givers & DD & ID, ASD, PD & Affiliate stigma & $\begin{array}{l}\text { Stigma was highest in care-givers of } \\
\text { children with ASD. }\end{array}$ \\
\hline $\begin{array}{l}\text { 33. Zablotsky, } 2013 \\
\text { (31) }\end{array}$ & 3 & $\begin{array}{l}\text { 1-item health } \\
\text { index \& } 3 \text {-item } \\
\text { stress index }\end{array}$ & Previous study & US & $\begin{array}{l}1,014 \text { cases } \& 55 \\
55,533 \text { controls }\end{array}$ & ASD, no ASD & $\begin{array}{l}\text { Stress (3-item } \\
\text { index) } \\
\text { Supports (5-item } \\
\text { index) }\end{array}$ & $\mathrm{MH}$ & $\begin{array}{l}\text { - Mothers of children with } \\
\text { ASD were more than } 7 \text { times } \\
\text { more likely to have high } \\
\text { levels of stress \& nearly } 3 \\
\text { times as likely to have } \\
\text { poorer MH } \\
\text { - Increased support associated with } \\
\text { better MH }\end{array}$ \\
\hline
\end{tabular}

ABC, Aberrant Behavior Checklist; AD, autistic disorder; AFS, Affiliate Stigma Scale; Array-based comparative genomic hybridization, aCGH; Autistic disorder; ASD, Autism spectrum disorder; ASD/ID, ASD or ID; ASQ, Autism Spectrum Quotient; BAP, Broad Autism Phenotype; BASC-2, Behaviour Assessment System for Children, Second Edition;BBCS, Bracken Basic Concept Scale; BDI, Beck Depression Inventory; carer, care-giver; CBC, Child Behaviour Checklist;CES-D, Center for Epidemiological Studies-Depression Scales; DAS, Dyadic Adjustment Scale; DBC, Developmental Behaviour Checklist;DBC-P, DBC-Primary Carer Version; DD, developmental disability, disorder or delay; dis, disability; DS, Down syndrome; DSSI, Delusions Symptoms States lnventory; ; FAD, MacMaster Family Activity Device; FQoL, Family QoL; FSI, Family Support Index; GDT, Gessel's Drawing Test; GFS, General Functioning Scale;GHQ-12, General Health Questionnaire-12-item version; GHQ-28, General Health Questionnaire-28-item version; HFASD, High-functioning ASD; ID, intellectual disability; Level, Level of evidence; MCS, Mental Component Summary of SF-36; MH, Mental health; MIS, Malin's Intelligence Scale; MSPSS, Multidimensional Scale of Perceived Social Support;MOS-SSS,Medical Outcome Study-Social Support Survey; NCBRF, Nisonger Child Behaviour Rating Form; neuro, neurological; NVS, Naming Vocabulary Subscale; PCS, Physical Component Summery of SF-36; PH, Physical health; pop, population; PSS, Parental Stress Scale; OR, odds ratio; PD, physical disabilities; PDD, Pervasive development disorder; PSI, Parenting Stress Index; PSI-SF, PSI: Short Form; QoL; Quality of Life; RR, risk ratio; SBS, Social Burden Scale; SDQ, Strengths and Difficulties Questionnaire 4-10 years: version for Australia; SF-12, 12-Item Short Form Health Survey; SF-36, 36-Item Short Form Health Survey;SFB, Seguin Form Board; SFS, Support Functions Scale; S-T-A-I, State-Trait-Anxiety-Inventory: German form; TD, typically developing; TDRWQ, Transition Daily Rewards and Worries Questionnaire; VSMS, Vineland Social Maturity Scale; WeeFIM-m, Functional Independence Measure for Children: modified; WHOQOL-BREF, World Health Organization Quality of Life-Brief. 
on parents with a child with ID [31-34]. Using the SF-12, an Australian study [35] provided substantial evidence (as defined in Table 1) that better mental health in parents older than 60 years was associated with having a partner and a large and supportive network of family, friends and neighbours. Others [36] used the Beck Depression Inventory and reported that single mothers living with a child with ID were more depressed than similar mothers living with a partner. There is also substantial evidence that poorer physical health in female care-givers of adults with developmental disabilities was associated with a lack of services [37] and that the effect of stress on physical health was moderated by parental social support [38].

\section{Socio-economic status}

Higher socio-economic status (SES) was a protective factor for parental health and particularly maternal health [39-41]. For example, correlational analyses showed that parents of children with ASD with higher incomes had less distress and an improved QoL compared with those of lower incomes [41]. Similarly, there was strong evidence that socio-economically disadvantaged mothers had a greater risk of a psychiatric disorder than socio-economically advantaged mothers with a child with a similar disability $[39,40]$.

\section{Rewards and stigma}

In a comparison of mothers of children with Down syndrome $(n=21)$ and mothers of children with ASD $(n=17)$, researchers provided some evidence of greater attachment and gratification in the mothers of children with Down syndrome [42]. A case-control study, with a lower evidence-base [43], reported that mothers of children with Down syndrome felt more rewarded by parenting than mothers of children with other developmental disabilities.

Affiliate stigma (or self-stigmatization) was higher among care-givers of persons with ASD compared to care-givers of persons with ID or physical disabilities although the level of evidence was not strong [44]. There was also evidence that there was additional societal stigma towards the care-givers of children who appear normal but manifest abnormal behaviours [45].

\section{Diagnostic issues}

Diagnostic uncertainty and an unclear prognosis are factors which can cause the greatest concern to care-givers of children with ASD and those for whom a cause of their child's ID has not been identified [45-47]. The first of these studies [45] used a combination of interviews or focus groups with caregivers and the second [46] provided qualitative evidence by interviewing care-givers. In the third [47], German researchers compared the mothers of children with Down syndrome, ID of unknown cause and typically developing children with respect to anxiety, guilt and emotional burden using the State-Trait-Anxiety-Inventory and the Balanced Inventory of
Desirable Responding. They concluded that uncertainty was a major cause of stress in mothers of children with ID of unknown cause [47]. A fourth study used the UIm Quality of Life Inventory for Parents to examine the QoL of parents of a child with a developmental disability before genetic testing and after receiving a genetic cause for their child's disability. The results provided strong evidence that maternal QoL was improved once the underlying diagnosis was known [48].

\section{Broad Autism Phenotype}

The concept of mild autistic traits in the relatives of a person with ASD is termed the Broad Autism Phenotype [49]. Validated questionnaires such as the Autism Spectrum Quotient [50] and the Broad Autism Phenotype Questionnaire [51] are used to quantify the extent of this trait on a linear scale. Two research groups $[30,52]$ provided mild and weak levels of evidence that persons exhibiting the Broad Autism Phenotype were at an increased risk of depression.

\section{Maternal health by the child's diagnosis or sub-type and the impact of risk factors}

In a previous review [12], we found that mothers of children with Down syndrome generally had better health than mothers of children with other intellectual disabilities. In turn, mothers of children with intellectual disabilities other than Down syndrome, generally had better health than mothers of children with ASD [especially ASD without ID] (Figure 1).

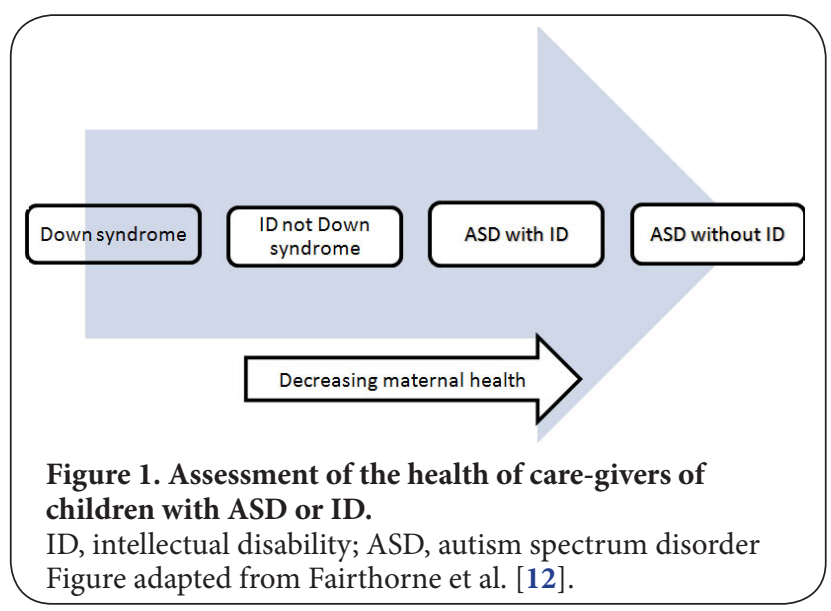

The disparities in the health of care-givers of children with ASD or ID may be partially explained by child characteristics associated with the particular diagnostic category. For example, challenging behaviours occur more often and are usually more severe in children with ASD than children with Down syndrome [53]. Thus, a commonly cited risk factor for poorer care-giver health and QoL outcomes, challenging child behaviour, is more often associated with ASD than Down syndrome. The increased likelihood of parents of children with ASD manifesting a Broad Autism Phenotype and its association with depression $[30,52]$ could explain why poorer 
Fairthorne et al., Journal of Autism 2018,

http://www.hoajonline.com/journals/pdf/2054-992X-5-1.pdf

doi: 10.7243/2054-992X-5-1

mental health is more common in parents of children with ASD than in parents of children with other developmental disabilities. Furthermore, personality type influences a person's willingness to seek support [54]. Hence, the lower social support evidenced in families with a child with ASD could be mediated by parental personality traits which are associated with the Broad Autism Phenotype. The consequent overall reduction in social support could thereby further increase the risk of poorer mental health in these parents. Moreover, the lag-time to diagnosis in comparison to Down syndrome, where children are diagnosed at birth, could also impact negatively on the mental health of parents of children with ASD. Furthermore, mothers of children with Down syndrome mostly reported that parenting was more fulfilling than did mothers of children with other disorders [42]. In contrast, caregivers of children with ASD were the most negatively affected by stigma [44], a risk factor for care-giver health. Moreover, Down syndrome is the most common genetic form of ID and children are readily recognised and supported by members of the community due to their distinctive phenotype [55]. On the other hand, children with ASD have no distinguishing phenotype and mostly appear physically normal to community bystanders [56]. Thus, there is the expectation that their behaviour would be normal which may not be the case [45].

\section{How risk and protective factors might impact care- giver health}

We have identified a total of seven risk or protective factors for health and QoL in the care-givers of children with ASD or ID. The impact of challenging child behaviour seems likely to be mediated by the increased parental stress generated by antisocial, and the sometimes dangerous and self-limiting behaviours in the child [57]. Challenging antisocial child behaviours such as running away and self-injurious behaviour such as head-banging can also severely limit family activities and thereby increase family social isolation [58]. Additionally, antisocial behaviour, when unexpected by the outsider, could further exacerbate the stigmatisation of parents [59]. Increased levels of extended family support and other support given to parents and their family were positively associated with parental well-being $[\mathbf{2 0 , 3 0 , 3 1 ]}$. Support provides relief for care-givers and in so doing provides opportunities for respite and thus engagement in social activities which might normally be reduced in care-givers of children with ASD or ID [54]. Socio-economic status may further impact on care-giver health since only well-resourced families may be able to gain support by enlisting paid care-givers and respite. Research has also found that families of lower SES have less knowledge of available support [60] and this may be because they are not equipped with the skills or assertiveness to navigate the systems.

Parents of children without a diagnosis for their disability can be disadvantaged [61] by the resultant lack of anticipatory and reproductive guidance, along with support by networking with parents of children with a similar diagnosis. Furthermore, these parents may feel ongoing pressure to search for a cause for their child's condition when either there is no clear aetiology or a lack of available technology to test a plausible diagnosis [47]. For example, the CDKL5 disorder is a genetic condition causing severe neuro-developmental impairment and refractory epilepsy from infancy [62]. Unfortunately, in Western Australia (along with many other cities in the Western world), genetic testing to confirm a diagnosis is not currently available. Such issues might negatively impact care-giver health.

\section{Summary and implications}

Care-givers of children with ASD are more likely to experience many of the risk factors and less likely to experience the protective factors we identified as contributing to poorer mental health. Interventions to reduce risk factors and increase protective factors could pave the way for improved care-giver health. For example, workshops to train parents to better manage challenging behaviours in their children and earlier diagnosis of ASD (and particularly ASD without ID) would seem likely to improve parental wellbeing. Similarly, helping to expand support networks might reduce care-giver burden and stress leading to improved health. High SES is a protective factor for care-giver health. Hence, increased subsidised supports for those of low SES, such as care-giver respite and home help, might also assist care-givers of children with ASD or ID to maintain their health.

\section{Competing interests}

The authors declare that they have no competing interests.

\section{Authors' contributions}

\begin{tabular}{|l|c|c|c|}
\hline Authors' contributions & JF & YM & HL \\
\hline Research concept and design & $\checkmark$ & -- & -- \\
\hline Collection and/or assembly of data & $\checkmark$ & -- & -- \\
\hline Data analysis and interpretation & $\checkmark$ & -- & -- \\
\hline Writing the article & $\checkmark$ & -- & -- \\
\hline Critical revision of the article & $\checkmark$ & $\checkmark$ & $\checkmark$ \\
\hline Final approval of article & $\checkmark$ & $\checkmark$ & $\checkmark$ \\
\hline Statistical analysis & -- & -- & -- \\
\hline
\end{tabular}

Publication history

Editor: Mohammad H. Hadadzadeh, Wheeling Jesuit University, USA. Received: 12-Oct-2017 Final Revised: 12-Jan-2018

Accepted: 20-Feb-2018 Published: 03-Mar-2018

\section{References}

1. American Psychiatric Association. Diagnostic and statistical manual of mental disorders American Psychiatric Publishing. 2013.

2. Fairthorne JC. Mothers of children with intellectual disability or autism spectrum disorder: pre-existing differences, health and quality of life. 2014.

3. Maulik PK, Mascarenhas MN, Mathers CD, Dua T and Saxena S. Prevalence of intellectual disability: a meta-analysis of populationbased studies. Res Dev Disabil. 2011; 32:419-36. | Article I PubMed 
Fairthorne et al., Journal of Autism 2018,

http://www.hoajonline.com/journals/pdf/2054-992X-5-1.pdf

4. Post MW. Definitions of quality of life: what has happened and how to move on. Top Spinal Cord Inj Rehabil. 2014; 20:167-80. | Article | PubMed Abstract | PubMed FullText

5. Miodrag $\mathrm{N}$ and Hodapp RM. Chronic stress and health among parents of children with intellectual and developmental disabilities. Curr Opin Psychiatry. 2010; 23:407-11. | Article | PubMed

6. Totsika V, Hastings RP, Emerson E, Lancaster GA and Berridge DM. A population-based investigation of behavioural and emotional problems and maternal mental health: associations with autism spectrum disorder and intellectual disability. J Child Psychol Psychiatry. 2011; 52:91-9. | Article | PubMed

7. Allik $\mathrm{H}$, Larsson $\mathrm{JO}$ and Smedje $\mathrm{H}$. Health-related quality of life in parents of school-age children with Asperger Syndrome or High-Functioning Autism. Health Qual Life Outcomes. 2006; 4:1. | Article | PubMed Abstract | PubMed FullText

8. Singer GH. Meta-analysis of comparative studies of depression in mothers of children with and without developmental disabilities. Am J Ment Retard. 2006; 111:155-69. | Article | PubMed

9. Fairthorne J, Jacoby P, Bourke J, de Klerk $\mathrm{N}$ and Leonard H. Onset of maternal psychiatric disorders after the birth of a child with autism spectrum disorder: A retrospective cohort study. Autism. 2016; 20:3744. | Article | PubMed

10. Fairthorne J, Jacoby P, Bourke J, de Klerk N and Leonard H. Onset of maternal psychiatric disorders after the birth of a child with intellectual disability: a retrospective cohort study. J Psychiatr Res. 2015; 61:223-30. | Article | PubMed

11. Giallo R, Wood CE, Jellett $R$ and Porter R. Fatigue, wellbeing and parental self-efficacy in mothers of children with an autism spectrum disorder. Autism. 2013; 17:465-80. | Article | PubMed

12. Fairthorne J, de Klerk $\mathrm{N}$ and Leonard $\mathrm{H}$. Health of mothers of children with intellectual disability or autism spectrum disorder: a review of the literature. Medical Research Archives. 2015; 3:1-21.

13. Piven J, Chase GA, Landa R, Wzorek M, Gayle J, Cloud D and Folstein S. Psychiatric disorders in the parents of autistic individuals. J Am Acad Child Adolesc Psychiatry. 1991; 30:471-8. | Article | PubMed

14. Maternal depression and child development. Paediatr Child Health. 2004; 9:575-598. | PubMed Abstract | PubMed FullText

15. Walker SP, Wachs TD, Gardner JM, Lozoff B, Wasserman GA, Pollitt E and Carter JA. Child development: risk factors for adverse outcomes in developing countries. Lancet. 2007; 369:145-57. | Article | PubMed

16. West S, King V, Carey T, Lohr K, McKoy N and Sutton S et al. Systems to rate the strength of scientific evidence. evidence report/technology assessment no. 47 Evidence Report/Technology Assessment No 47 (Prepared by the Research Triangle Institute-University of North Carolina Evidence-based Practice Center under Contract No 290-970011). 2002.

17. Dillon SR, Adams D, Goudy L, Bittner M and McNamara S. Evaluating Exercise as Evidence-Based Practice for Individuals with Autism Spectrum Disorder. Front Public Health. 2016; 4:290. | Article | PubMed Abstract | PubMed FullText

18. Emerson E. Mothers of children and adolescents with intellectual disability: social and economic situation, mental health status, and the self-assessed social and psychological impact of the child's difficulties. $J$ Intellect Disabil Res. 2003; 47:385-99. | Article | PubMed

19. Bourke J, Ricciardo B, Bebbington A, Aiberti K, Jacoby P, Dyke P, Msall $\mathrm{M}$, Bower $\mathrm{C}$ and Leonard $\mathrm{H}$. Physical and mental health in mothers of children with Down syndrome. J Pediatr. 2008; 153:320-6. | Article | PubMed Abstract | PubMed FullText

20. Bromley J, Hare DJ, Davison K and Emerson E. Mothers supporting children with autistic spectrum disorders: social support, mental health status and satisfaction with services. Autism. 2004; 8:409-23. | Article | PubMed

21. Estes A, Munson J, Dawson G, Koehler E, Zhou XH and Abbott R. Parenting stress and psychological functioning among mothers of preschool children with autism and developmental delay. Autism. 2009; 13:375-87. | Article | PubMed Abstract | PubMed FullText
22. Firth I and Dryer R. The predictors of distress in parents of children with autism spectrum disorder. J Intellect Dev Disabil. 2013; 38:163-71. | Article I PubMed

23. Herring S, Gray K, Taffe J, Tonge B, Sweeney D and Einfeld S. Behaviour and emotional problems in toddlers with pervasive developmental disorders and developmental delay: associations with parental mental health and family functioning. J Intellect Disabil Res. 2006; 50:874-82. | Article | PubMed

24. McStay RL, Trembath D and Dissanayake C. Stress and family quality of life in parents of children with autism spectrum disorder: parent gender and the double ABCX model. J Autism Dev Disord. 2014; 44:3101-18. | Article | PubMed

25. Paynter J, Riley E, Beamish W, Davies M and Milford T. The double $A B C X$ model of family adaptation in families of a child with an autism spectrum disorder attending an Australian early intervention service. Research in Autism Spectrum Disorders. 2013; 7:1183-95.

26. Eisenhower A, Blacher J and Baker BL. Mothers' perceived physical health during early and middle childhood: relations with child developmental delay and behavior problems. Res Dev Disabil. 2013; 34:1059-68. | Article | PubMed Abstract | PubMed FullText

27. Harvey JM, O'Callaghan MJ and Vines B. Prevalence of maternal depression and its relationship to ADL skills in children with developmental delay. J Paediatr Child Health. 1997; 33:42-6. | Article | PubMed

28. Baghdadli A, Pry R, Michelon C and Rattaz C. Impact of autism in adolescents on parental quality of life. Qual Life Res. 2014; 23:1859-68. | Article | PubMed

29. Ji B, Zhao I, Turner C, Sun M, Yi R and Tang S. Predictors of health-related quality of life in Chinese caregivers of children with autism spectrum disorders: a cross-sectional study. Arch Psychiatr Nurs. 2014; 28:327-32. | Aritcle | PubMed

30. Ingersoll B, Meyer K and Becker MW. Increased rates of depressed mood in mothers of children with ASD associated with the presence of the broader autism phenotype. Autism Res. 2011; 4:143-8. | Article | PubMed

31. Zablotsky B, Bradshaw CP and Stuart EA. The association between mental health, stress, and coping supports in mothers of children with autism spectrum disorders. J Autism Dev Disord. 2013; 43:1380-93. | Article | PubMed

32. Schieve LA, Boulet SL, Kogan MD, Yeargin-Allsopp M, Boyle CA, Visser SN, Blumberg SJ and Rice C. Parenting aggravation and autism spectrum disorders: 2007 National Survey of Children's Health. Disabil Health J. 2011; 4:143-52. | Article | PubMed

33. Mugno D, Ruta L, D'Arrigo VG and Mazzone L. Impairment of quality of life in parents of children and adolescents with pervasive developmental disorder. Health Qual Life Outcomes. 2007; 5:22. | Article | PubMed Abstract | PubMed FullText

34. Montes $G$ and Halterman JS. Psychological functioning and coping among mothers of children with autism: a population-based study. Pediatrics. 2007; 119:e1040-6. | Article | PubMed

35. Llewellyn G, McConnell D, Gething L, Cant R and Kendig H. Health status and coping strategies among older parent-carers of adults with intellectual disabilities in an Australian sample. Res Dev Disabil. 2010; 31:1176-86. | Article | PubMed

36. Olsson MB and Hwang CP. Depression in mothers and fathers of children with intellectual disability. J Intellect Disabil Res. 2001; 45:535-43. I Article | PubMed

37. Caldwell J. Consumer-directed supports: economic, health, and social outcomes for families. Ment Retard. 2006; 44:405-17. | Article | PubMed

38. Cantwell J, Muldoon OT and Gallagher S. Social support and mastery influence the association between stress and poor physical health in parents caring for children with developmental disabilities. Res Dev Disabil. 2014; 35:2215-23. | Article | PubMed

39. Emerson E, McCulloch A, Graham H, Blacher J, Llwellyn GM and Hatton C. Socioeconomic circumstances and risk of psychiatric disorders among 
Fairthorne et al., Journal of Autism 2018,

parents of children with early cognitive delay. Am J Intellect Dev Disabil. 2010; 115:30-42. | Article | PubMed

40. Singhi PD, Goyal L, Pershad D, Singhi S and Walia BN. Psychosocial problems in families of disabled children. Br J Med Psychol. 1990; 63 ( Pt 2):173-82. I PubMed

41. Dardas LA and Ahmad MM. Psychosocial correlates of parenting a child with autistic disorder. J Nurs Res. 2014; 22:183-91. | Article | PubMed

42. Hoppes $K$ and Harris S. Perceptions of child attachment and maternal gratification in mothers of children with autism and Down syndrome. Journal of Clinical Child Psychology. 1990; 19:365-70.

43. Corrice AM and Glidden LM. The Down syndrome advantage: fact or fiction? Am J Intellect Dev Disabil. 2009; 114:254-68. | Article | PubMed

44. Werner $S$ and Shulman C. Does type of disability make a difference in affiliate stigma among family caregivers of individuals with autism, intellectual disability or physical disability? J Intellect Disabil Res. 2015 59:272-83. | Article | PubMed

45. Kelso T, French D and Fernandez M. Stress and coping in primary caregivers of children with a disability: a qualitative study using the Lazarus and Folkman Process Model of Coping. Journal of Research in Special Educational Needs. 2005; 5:3-10.

46. DeGrace B, Hoffman C, Hutson T and Kolobe T. Families' experiences and occupations following the diagnosis of autism. Journal of Occupational Science. 2014; 21:309-21. | Article

47. Lenhard W, Breitenbach E, Ebert H, Schindelhauer-Deutscher HJ and Henn W. Psychological benefit of diagnostic certainty for mothers of children with disabilities: lessons from Down syndrome. Am J Med Genet A. 2005; 133A:170-5. I Article I PubMed

48. Lingen M, Albers L, Borchers M, Haass S, Gartner J, Schroder S, Goldbeck $\mathrm{L}$, von Kries $\mathrm{R}$, Brockmann $\mathrm{K}$ and Zirn $\mathrm{B}$. Obtaining a genetic diagnosis in a child with disability: impact on parental quality of life. Clin Genet. 2016; 89:258-66. | Article | PubMed

49. Losh M, Adolphs R, Poe MD, Couture S, Penn D, Baranek GT and Piven J. Neuropsychological profile of autism and the broad autism phenotype. Arch Gen Psychiatry. 2009; 66:518-26. | Article | PubMed Abstract | PubMed FullText

50. Bishop DV, Maybery M, Maley A, Wong D, Hill W and Hallmayer J. Using self-report to identify the broad phenotype in parents of children with autistic spectrum disorders: a study using the Autism-Spectrum Quotient. J Child Psychol Psychiatry. 2004; 45:1431-6. I Article I PubMed

51. Hurley RS, Losh M, Parlier M, Reznick JS and Piven J. The broad autism phenotype questionnaire. J Autism Dev Disord. 2007; 37:1679-90. | Article | PubMed

52. Ingersoll B, Meyer K and Becker MW. Increased rates of depressed mood in mothers of children with ASD associated with the presence of the broader autism phenotype. Autism Res. 2011; 4:143-8. | Article | PubMed

53. Griffith GM, Hastings RP, Nash S and Hill C. Using matched groups to explore child behavior problems and maternal well-being in children with Down syndrome and autism. J Autism Dev Disord. 2010; 40:610-9. | Article | PubMed

54. Boyd BA. Examining the relationship between stress and lack of social support in mothers of children with autism. Focus on Autism and Other Developmental Disabilities. 2002; 17:208-15.

55. Holtzman D and Epstein CJ. The molecular genetics of Down syndrome. In: Friedmann T, editor. Molecular Genetic Medicine. 2. San Diego, California: Academic Press; 2013; 105-20.

56. Higgins DJ, Bailey SR and Pearce JC. Factors associated with functioning style and coping strategies of families with a child with an autism spectrum disorder. Autism. 2005; 9:125-37. I Article I PubMed

57. Hastings RP. Parental stress and behaviour problems of children with developmental disability. Journal of Intellectual and Developmental Disability. 2002; 27:149-60.

58. Woodgate RL, Ateah C and Secco L. Living in a world of our own: the experience of parents who have a child with autism. Qual Health Res. 2008; 18:1075-83. | Article | PubMed

59. Gray DE. 'Everybody just freezes. Everybody is just embarrassed': Felt and enacted stigma among parents of children with high functioning autism. Sociology of Health and IIIness. 24:734-49.

60. Douma JC, Dekker MC and Koot HM. Supporting parents of youths with intellectual disabilities and psychopathology. J Intellect Disabil Res. 2006; 50:570-81. | Article I PubMed

61. Watson SL, Hayes SA and Radford-Paz E. Diagnose me please!": A review of research about the journey and initial impact of parents seeking a diagnosis of developmental disability for their child. International review of research in developmental disabilities. 2011; 41:31-72.

62. Tao J, Van Esch H, Hagedorn-Greiwe M, Hoffmann K, Moser B, Raynaud M, Sperner J, Fryns JP, Schwinger E, Gecz J, Ropers HH and Kalscheuer VM. Mutations in the X-linked cyclin-dependent kinase-like 5 (CDKL5/STK9) gene are associated with severe neurodevelopmental retardation. Am J Hum Genet. 2004; 75:1149-54. | Article I PubMed Abstract | PubMed FullText

63. Lach LM, Kohen DE, Garner RE, Brehaut JC, Miller AR, Klassen AF and Rosenbaum PL. The health and psychosocial functioning of caregivers of children with neurodevelopmental disorders. Disabil Rehabil. 2009; 31:607-18. | Article | PubMed

\section{Citation:}

Fairthorne J, Mori Y and Leonard H. Risk and protective factors for the health of primary care-givers of children with autism spectrum disorders or intellectual disability: a narrative review. J Autism. 2018; 5:1. http://dx.doi.org/10.7243/2054-992X-5-1 